\title{
Coupled cluster benchmarks of water monomers and dimers extracted from DFT liquid water: the importance of monomer deformations
}

\author{
Biswajit Santra $^{1}$, Angelos Michaelides ${ }^{1,2}$ 田 and Matthias Scheffler ${ }^{1}$ \\ ${ }^{1}$ Fritz-Haber-Institut der Max-Planck-Gesellschaft, Faradayweg 4-6, 14195 Berlin, Germany \\ ${ }^{2}$ London Centre for Nanotechnology and Department of Chemistry, \\ University College London, London WC1E 6BT, UK
}

\begin{abstract}
To understand the performance of popular density-functional theory (DFT) exchange-correlation (xc) functionals in simulations of liquid water, water monomers and dimers were extracted from a PBE simulation of liquid water and examined with coupled cluster with single and double excitations plus a perturbative correction for connected triples $[\mathrm{CCSD}(\mathrm{T})]$. $\operatorname{CCSD}(\mathrm{T})$ reveals that most of the dimers are unbound compared to two gas phase equilibrium water monomers, largely because monomers within the liquid have distorted geometries. Of the three xc functionals tested, PBE and BLYP systematically underestimate the cost of the monomer deformations and consequently predict too large dissociation energies between monomers within the dimers. This is in marked contrast to how these functionals perform for an equilibrium water dimer and other small water clusters in the gas phase, which only have moderately deformed monomers. PBE0 reproduces the CCSD $(\mathrm{T})$ monomer deformation energies very well and consequently the dimer dissociation energies much more accurately than PBE and BLYP. Although this study is limited to water monomers and dimers, the results reported here may provide an explanation for the overstructured radial distribution functions routinely observed in BLYP and PBE simulations of liquid water and are of relevance to water in other phases and to other associated molecular liquids.
\end{abstract}

\section{Introduction}

Density-functional theory (DFT) has been widely used to study liquid water. However, how well DFT with popular exchange-correlation (xc) functionals such as PBE 1] and BLYP [2, 3] performs in describing the structural and dynamic properties of liquid water is a matter of more than a little contention. The debates, which are numerous, have hinged on issues such as the radial distribution functions (RDFs) (in particular the $\mathrm{O}-\mathrm{O}$ and $\mathrm{O}-\mathrm{H}$ RDFs), diffusion coefficient, and average number of hydrogen bonds (HBs).

It is now clear that most standard DFT molecular dynamics (MD) simulations with PBE and BLYP predict an overstructured RDF compared to experiment. By overstructured, we mainly mean that the first peak in the O-O RDF (referred to as $g_{\mathrm{O}-\mathrm{O}}^{\max }$ ) is higher than experiment. Consequently the computed diffusion coefficient is too small and the average number of HBs too large. Extended discussions on the magnitude and origin of the overstructuring can be found in e.g. Refs. $4,5,6,6,7,8,9,9$, $10,11,12,13,14,15,16,17,18,19,20,21,22,23,24,25]$. In brief, some of the relevant factors include: (i) The intrinsic error associated with a given xc functional (including an improper account of van der Waals forces 18, 26 ); (ii) The omission of quantum nuclear effects 27, 28, 29, 30, 31]; and (iii) The simulation protocol, with relevant factors in this regard being: (a) number of water molecules in the simulation cell [16]; (b) the density of the water within the cell [7, 8, 9]; (c) basis set [10, 32]; (d) fictitious electron mass in Car-Parrinello MD simula-

*Electronic address: angelos.michaelides@ucl.ac.uk tions [33]; and so on. Since the first DFT MD simulation of liquid water in 1993 34], important strides have been made to understand how each of the above factors impact upon the computed properties of liquid water. However, simultaneously addressing all issues that could account for the difference between the experimental and theoretical RDFs and diffusion coefficients is not practicable, not to mention the uncertainties that are present in the experimental data itself [35, 36, 37]. Therefore, it has become common to attempt to shed light on the performance of DFT xc functionals for treating water by investigating well defined gas phase water clusters for which precise comparison can be made to high level quantum chemistry methods. This approach has been useful and allowed the intrinsic accuracy of many xc functionals to be precisely established $26,38,39,40,41,42,43,44,45$, 46, 47, 48, 49, 50, 51, 52], information that may be of relevance to liquid water.

With few exceptions [47, 48, 49, 50, 51], previous gas phase benchmark studies of water clusters have focussed on exploring equilibrium or other stationary point configurations of the gas phase intermolecular potential energy surfaces. However, in the liquid the structures of water clusters and even the water monomers themselves can be considerably different from those of gas phase clusters. For example, the distribution of intramolecular O$\mathrm{H}$ bond lengths in the liquid ranges from $\sim 0.75$ to $\sim 1.25$ $\AA$ 35, 37]. Yet in gas phase water clusters such as dimers to hexamers O-H bond lengths deviate by $<0.05 \AA$ from the equilibrium water monomer $\mathrm{O}-\mathrm{H}$ bond length of 0.96 $\AA 26,38$. Whether or not the performance of DFT $\mathrm{xc}$ functionals obtained from gas phase studies on water clusters holds for the 'deformed' structures present in the liquid (referred to throughout this article as 'deformed') remains an important open question. Indeed 
there is already evidence that the benchmark reference data obtained from gas phase clusters does not easily translate to the liquid. For example, BLYP predicts a dissociation energy for the equilibrium gas phase water dimer that is $35 \mathrm{meV}$ too small, yet at a water density of 1 $\mathrm{g} / \mathrm{cm}^{3}$ it predicts a $g_{\mathrm{O}-\mathrm{O}}^{\max }$ that is about $5 \%-15 \%$ too high. Similarly, PBE predicts the dimer dissociation energy to within $10 \mathrm{meV}$ precision, yet yields an even greater $g_{\mathrm{O}-\mathrm{O}}^{\max }$ than BLYP. Related to this, MD simulations of liquid water have shown that the computed $g_{\mathrm{O}-\mathrm{O}}^{\max }$ can be considerably reduced if the $\mathrm{O}-\mathrm{H}$ bonds in the water monomers in the liquid are held rigid at some predefined bond length. Specifically, Allesch et al. found that the PBE $g_{\mathrm{O}-\mathrm{O}}^{\max }$ decreased by $\sim 10 \%$ upon going from fully relaxed water monomers to a liquid with monomer $\mathrm{O}-\mathrm{H}$ bonds fixed at $\sim 1 \AA$ [53]. Likewise, Leung et al. have shown through a careful and systematic series of simulations that the length of the $\mathrm{O}-\mathrm{H}$ bonds for rigid water MD simulations directly correlates with $g_{\mathrm{O}-\mathrm{O}}^{\max }[54]$ : as the intramolecular $\mathrm{O}-\mathrm{H}$ bonds are allowed to lengthen, $g_{\mathrm{O}-\mathrm{O}}^{\max }$ increases.

The studies with rigid water and the realization that water monomer and cluster structures in the liquid are likely to differ considerably from gas phase water clusters has prompted us to assess the performance of DFT $\mathrm{xc}$ functionals on water structures more representative of those present in the liquid. To this end we report herein on the accuracy of three DFT xc functionals for various deformed monomers and dimers taken from a PBE simulation of liquid water. Two of the most popular generalized gradient approximation (GGA) $\mathrm{xc}$ functionals for liquid water simulations (PBE and BLYP) and one of the most accurate hybrid functionals for small water clusters (PBE0 [55]) are assessed here. As a reference, coupled cluster with single and double excitations plus a perturbative correction for connected triples $[\operatorname{CCSD}(\mathrm{T})]$ is used with energies extrapolated to the complete basis set limit (CBS). The CCSD(T) reference calculations reveal that $75 \%$ of the dimers extracted from within the first coordination shell of the liquid are unbound relative to two equilibrium (gas phase) water monomers. This is mainly due to the large deformation of the monomers inside the liquid compared to the gas phase equilibrium monomer structure. PBE and BLYP consistently underestimate the cost of the monomer deformation, specifically, O-H bond stretching. As a consequence, both PBE and BLYP systematically overbind the deformed dimers extracted from the liquid, by as much as 80 and $43 \mathrm{meV}$, respectively. These errors are much larger than the usual errors associated with these $\mathrm{xc}$ functionals for the gas phase equilibrium dimer [38]. In general, the performance of PBE0 is superior to the two GGAs but noticeable errors are identified for all functionals including PBE0 for the particularly long $\mathrm{O}-\mathrm{H}$ bonds encountered at the shortest O-O separations. Although this study is restricted to monomers and dimers (and in a sense resembles a highly limited cluster expansion study of the liquid), the results reported here provide a possible explanation for the overstructured

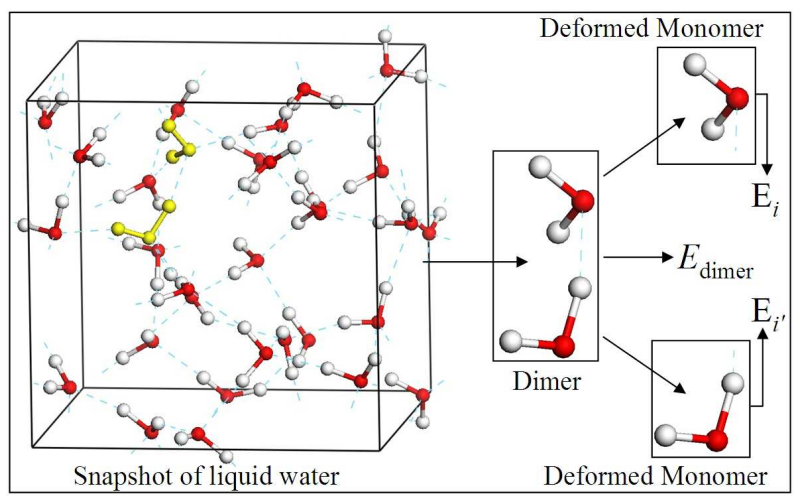

FIG. 1: (Color online) From a PBE MD simulation of liquid water, water dimers are extracted (e.g. the highlighted dimer in yellow). Single point energy calculations are then performed with CCSD(T), PBE, BLYP, and PBE0 on the deformed dimers $\left(E_{\text {dimer }}\right)$ and the constituent deformed monomers $\left(E_{i}, E_{i^{\prime}}\right)$. These energies are then used to evaluate the electronic dissociation energy of the dimers (Eqn. (4)) and the associated 1-body (Eqn. (2)) and 2-body (Eqn. (3)) energies. The deformation of the monomers compared to a gas phase equilibrium monomer is also quantified with (Eqn. (1)).

RDFs routinely observed in BLYP and PBE simulations of liquid water. The significance of these results to water in other phases and to other associated molecular liquids is also briefly discussed.

\section{Methods, procedures, and definition of parameters}

Several methods have been employed to study the water clusters examined here. We now briefly describe some of the relevant computational details, how the water monomers and dimers are selected from the liquid, and then define the energetic and structural parameters used in the subsequent analysis.

\section{A. Liquid water}

To generate water monomer and dimer structures representative of those present in liquid water a BornOppenheimer molecular dynamics simulation of $32 \mathrm{D}_{2} \mathrm{O}$ molecules in a periodic cubic box of length $9.8528 \AA$ was performed with the CPMD code [56]. The PBE xc functional was used along with hard pseudopotentials of Goedecker et al. [57] and an associated plane wave energy cut-off of 125 Ry. This simulation was run for $30 \mathrm{ps}$ with an integration time step of 0.5 fs. A Nosé-Hoover chain thermostat was used to maintain a target temperature of $330 \mathrm{~K}$.

Water monomers and dimers were then extracted from the MD simulation. To get an uncorrelated sample of structures, 6-7 dimers were selected each 2 ps over the last $20 \mathrm{ps}$ of the MD trajectory. In total 66 

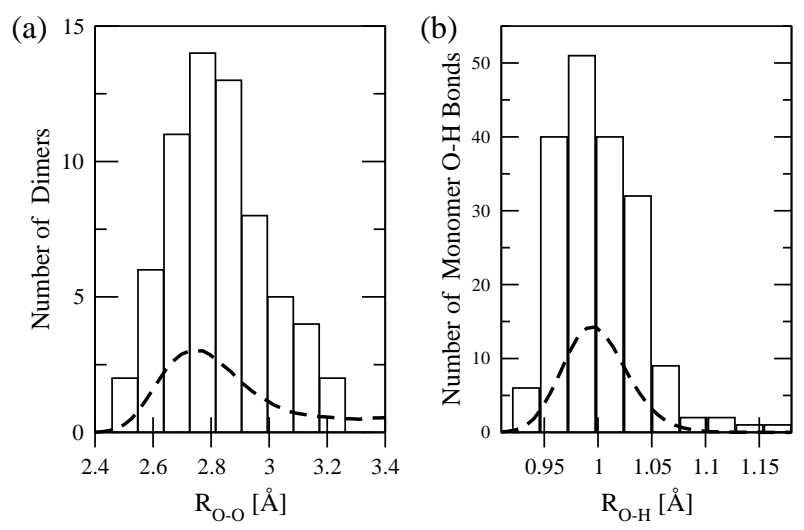

FIG. 2: (a) Distribution of the number of water dimers selected from the PBE MD simulation of liquid water (plotted as bars) as a function of the O-O separations $\left(R_{O-O}\right)$ within the dimer. (b) Distribution of the number of O-H bonds for the water monomers selected from the liquid (plotted as bars). The dashed lines represent the corresponding O-O and O$\mathrm{H}$ RDFs from the same PBE liquid water simulation. Only dimers within the first coordination shell of liquid water are considered here.

bonded dimers were selected (comprising 92 individual monomers). The criteria we followed for selecting dimers were that: (i) they were from within the first coordination shell of the O-O RDF, i.e., all chosen $\mathrm{O}-\mathrm{O}$ distances $\left(R_{O-O}\right)$ are $\leq 3.4 \AA$; and (ii) the distribution of all $66 R_{O-O}$ of the dimers resembles the O-O RDF for the first coordination shell. Fig. 2(a) illustrates that the distribution of dimers selected does indeed resemble the computed O-O RDF of our MD simulation reasonably well. As an independent check we note that the distribution in the values of the intra-molecular $\mathrm{O}-\mathrm{H}$ bond lengths associated with all the selected water molecules is also in reasonably good agreement with the first peak of our computed $\mathrm{O}-\mathrm{H}$ RDF of liquid water [Fig. 2(b)].

\section{B. Clusters}

The PBE water monomers and dimers extracted from the liquid water simulation were then examined with a few DFT xc functionals and $\operatorname{CCSD}(\mathrm{T})$. Throughout this study structures collected from the liquid water simulations are used for single point energy calculations with the various methods and no geometries are optimized, unless explicitly stated otherwise. The DFT calculations on the gas phase water monomers and dimers were performed with the GAUSSIAN03 code [58], using large Dunning correlation consistent aug-cc-pV5Z basis sets [59]. We have shown before that for DFT xc functionals such as the ones considered here, this basis set is large enough to get dissociation energies within about 1 $\mathrm{meV} / \mathrm{H}_{2} \mathrm{O}$ of the CBS limit 38]. Results from just three $\mathrm{xc}$ functionals will be reported, specifically two GGAs that are widely employed in DFT simulations of liquid water (PBE and BLYP), and the hybrid PBE0 functional, which is one of the most accurate functionals in predicting the absolute dissociation energies of small gas phase water clusters (dimer to hexamer) [26, 38].

The $\operatorname{CCSD}(\mathrm{T})$ calculations were performed with the NWChem code [60] with localized Gaussian basis sets. Specifically, aug-cc-pVnZ basis sets $(\mathrm{n}=\mathrm{T}, \mathrm{Q}$, and 5$)$ were used and the resultant energies extrapolated to the complete basis set limit (CBS) with the same standard heuristic schemes as employed by us before [26, 38]. $\operatorname{CCSD}(\mathrm{T}) / \mathrm{CBS}$ is the theoretical 'gold standard' for systems of the size considered here and, in the following, differences between a given xc functional and $\operatorname{CCSD}(\mathrm{T})$ are referred to as errors with that xc functional. In total $>600 \operatorname{CCSD}(\mathrm{T})$ calculations have been performed for the reference data presented in this paper.

\section{Definition of Parameters}

In order to quantitatively compare the structure of the molecules extracted from the liquid to a gas phase equilibrium monomer we define a quantity $S_{d}$, the deformation, as,

$$
S_{d}=\sqrt{\sum_{N}\left(\mathbf{R}_{N}-\mathbf{r}_{N}\right)^{2}},
$$

where $N$ is the number of atoms, $\mathbf{R}$ and $\mathbf{r}$ denote the coordinate vectors of deformed and gas phase equilibrium monomer structures, respectively.

Several energy terms will appear repeatedly and it is also useful to define them here. The one-body energy $\left(E_{1 b}\right)$ of a water monomer is calculated as,

$$
E_{1 b}=E_{i}-E_{\text {equilibrium }}
$$

where $E_{\text {equilibrium }}$ is the energy of the gas phase water monomer at equilibrium and $E_{i}$ is the energy of a deformed monomer. The two-body energy $\left(E_{2 b}\right)$ of a dimer is defined as:

$$
E_{2 b}=E_{\text {dimer }}-E_{i}-E_{i^{\prime}},
$$

where $E_{\text {dimer }}$ is the total energy of the dimer. The electronic dissociation energy $\left(D_{e}\right)$ of the dimers is given by,

$$
D_{e}=E_{\text {dimer }}-2 \times E_{\text {equilibrium }} .
$$

Fig. 1 schematically illustrates each of the above energetic quantities and the overall procedure used in this study. Since the structures considered here have been taken from a PBE liquid water simulation, $E_{\text {equilibrium }}$ is calculated with a PBE structure [61]. The error conceded by DFT xc functionals $(\Delta E)$ in comparison to $\operatorname{CCSD}(\mathrm{T}) / \mathrm{CBS}$ is given as,

$$
\Delta E=E_{\mathrm{CCSD}(\mathrm{T})}-E_{\mathrm{DFT}}
$$



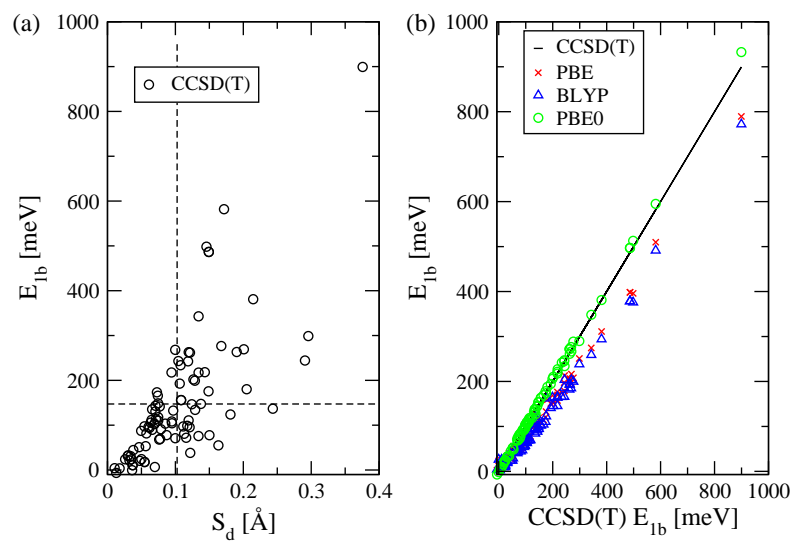

FIG. 3: (Color online) (a) $\operatorname{CCSD}(\mathrm{T})$ one-body energy $\left(E_{1 b}\right)$ versus deformation $\left(S_{d}\right)$ for water monomers taken from PBE liquid water. The horizontal and vertical dashed lines indicate the mean value of $E_{1 b}$ and the mean value of the deformation, respectively. (b) Comparison of $E_{1 b}$ of PBE, BLYP, and PBE0 with $\operatorname{CCSD}(\mathrm{T})$.

where $E_{\mathrm{CCSD}(\mathrm{T})}$ and $E_{\mathrm{DFT}}$ are energies obtained from $\operatorname{CCSD}(\mathrm{T}) / \mathrm{CBS}$ and DFT, respectively.

\section{Results}

Now we examine the water monomers extracted from the liquid, focusing on the cost to go from the gas phase equilibrium monomer structure to the deformed structures present in the liquid. Following this we consider the water dimers. In each case we compare the results of the various DFT xc functionals to the $\operatorname{CCSD}(\mathrm{T}) / \mathrm{CBS}$ references.

\section{A. Monomers}

To begin, $\operatorname{CCSD}(\mathrm{T})$ was used to establish the relative energies of the monomers taken from the liquid compared to the gas phase equilibrium monomer, i.e., $\operatorname{CCSD}(\mathrm{T})$ $E_{1 b}$ energies were computed for all 92 monomers. As can be seen from Fig. 3(a) these are distributed in a very large range from $\sim 0$ to $+900 \mathrm{meV}$ with a mean value of $+147 \mathrm{meV}$. Thus on average the monomers extracted from the liquid are $147 \mathrm{meV}$ less stable than the gas phase equilibrium monomer, a surprisingly large energy. In quantifying the amount of deformation (Eqn. 11) for each monomer we find, as expected, a general increase in $E_{1 b}$ with the extent of deformation [Fig. 3(a)]. The average deformation of the monomers is $\sim 0.1 \AA$, which gives us a measure of how deformed water monomers are in a PBE liquid water structure.

Now we consider how the deformation energies computed with PBE, BLYP, and PBE0 compare to $\operatorname{CCSD}(\mathrm{T})$. This is shown in Fig. 3(b), a parity plot of $E_{1 b}$ for the three xc functionals compared to $\operatorname{CCSD}(\mathrm{T})$. Immediately it can be seen that the performance of the
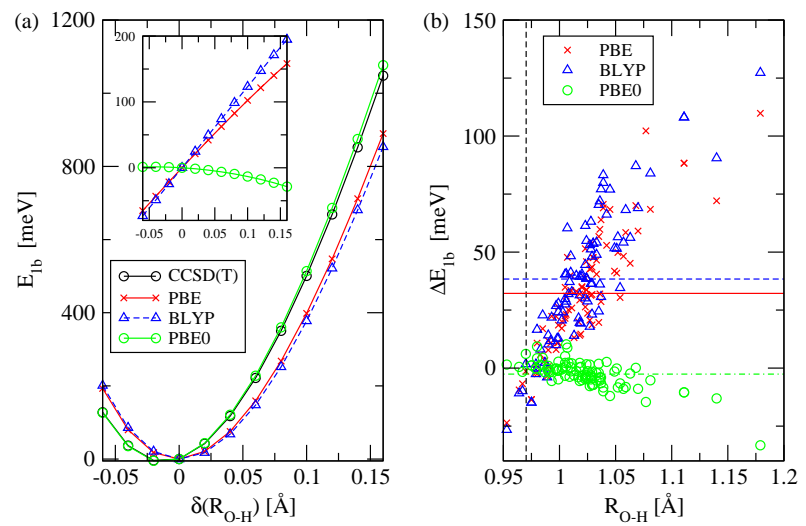

FIG. 4: (Color online) (a) Variation in the one-body energy $\left(E_{1 b}\right)$ with the $\mathrm{O}-\mathrm{H}$ bond lengths of a water monomer calculated with $\operatorname{CCSD}(\mathrm{T}), \mathrm{PBE}, \mathrm{BLYP}$, and PBE0. The inset shows the differences in $E_{1 b}$ of the three xc functionals compared to $\operatorname{CCSD}(\mathrm{T})$. (b) Errors in $E_{1 b}\left(\Delta E_{1 b}\right)$ for the deformed monomers selected from liquid water as a function of the longest $\mathrm{O}-\mathrm{H}$ bond of each monomer. The vertical dashed line indicates the gas phase equilibrium $\mathrm{O}-\mathrm{H}$ bond length $(0.97 \AA)$ of a monomer (optimized with PBE) and the horizontal solid, dashed, and dash-dotted lines represent the average errors of $\mathrm{PBE}, \mathrm{BLYP}$, and PBE0, respectively. Here, a positive error of $E_{1 b}$ indicates that it is too easy to stretch O-H bonds of the monomers with a given xc functional compared to $\operatorname{CCSD}(\mathrm{T})$.

GGAs is markedly different from the hybrid PBE0 functional. Specifically, for PBE and BLYP, $E_{1 b}$ is systematically too small compared to $\operatorname{CCSD}(\mathrm{T})$. On average the PBE and BLYP deformation energies are 32 and $38 \mathrm{meV}$, respectively, smaller than that obtained from $\operatorname{CCSD}(\mathrm{T})$. The size of the error simply increases with the total $\operatorname{CCSD}(\mathrm{T}) E_{1 b}$ [Fig. 3(b)] and for the largest deformations is on the order of $100 \mathrm{meV}$. Remembering that the monomer deformation is, of course, an endothermic process, smaller values of $E_{1 b}$ therefore indicate that it is too easy to deform monomers to their liquid structures with $P B E$ or $B L Y P$ compared to $C C S D(T)$. In contrast to the GGAs, PBE0 produces $E_{1 b}$ in excellent agreement with $\operatorname{CCSD}(\mathrm{T})$ with a mean error of only $-3 \mathrm{meV}$. This small negative error reveals that it is marginally too expensive to deform the monomers to their liquid water structure with PBE0 compared to CCSD(T). Since the only difference between PBE and PBE0 is the 25\% Hartree-Fock (HF) exchange in the latter, we conclude that the inclusion of exact exchange remedies the large error in $E_{1 b}$ almost completely. Why this is so will be discussed in section IV.

The monomers extracted from the liquid have both modified bond lengths and $\mathrm{H}-\mathrm{O}-\mathrm{H}$ internal angles. In order to understand in detail where the errors in $E_{1 b}$ for the GGAs come from we carried out a simple series of tests where bond lengths and the internal angle were varied independently. The tests show that the main error in the GGAs comes from the bond stretching. For example Fig. 4(a) shows that the symmetric stretching of the 
TABLE I: Computed harmonic vibrational frequencies for a water monomer. $\nu_{1}$ and $\nu_{2}$ are the asymmetric and symmetric $\mathrm{O}-\mathrm{H}$ stretching modes and $\nu_{3}$ is the $\mathrm{H}-\mathrm{O}-\mathrm{H}$ bending mode. All values are in $\mathrm{cm}^{-1}$ and calculated with an aug-cc-pVTZ basis set.

\begin{tabular}{cccc}
\hline \hline & $\nu_{1}$ & $\nu_{2}$ & $\nu_{3}$ \\
\hline CCSD(T) & 3921 & 3812 & 1648 \\
PBE & 3800 & 3696 & 1593 \\
BLYP & 3756 & 3655 & 1596 \\
PBE0 & 3962 & 3856 & 1633 \\
\hline \hline
\end{tabular}

$\mathrm{O}-\mathrm{H}$ bonds of a water monomer costs much less energy with PBE and BLYP compared to $\operatorname{CCSD}(\mathrm{T})$. The errors increase almost linearly with the stretching [inset Fig. [4(a)] and are as large as $\sim 200 \mathrm{meV}$ when the $\mathrm{O}-\mathrm{H}$ bonds are $0.16 \AA$ longer than the gas phase equilibrium bond length of $0.97 \AA$. A bond stretch of $0.16 \AA$ may sound like a lot but monomers with $\mathrm{O}-\mathrm{H}$ bonds even as long as $1.18 \AA$ are present in our PBE MD simulation and in experiment and in $a b$ initio path integral simulations even longer O-H bonds are observed [31, 37]. As we saw for the structures taken from the liquid, PBE0 is in very good agreement with $\operatorname{CCSD}(\mathrm{T})$ and even for the longest $\mathrm{O}-\mathrm{H}$ bond of $1.18 \AA$ comes within $34 \mathrm{meV}$ of $\operatorname{CCSD}(\mathrm{T})$. In addition alterations of the $\mathrm{H}-\mathrm{O}-\mathrm{H}$ angle were considered but this makes much less of a contribution to the error in the $\mathrm{xc}$ functionals than what we find for bond stretching. For example, increasing (decreasing) the bond angle by $15^{\circ}$ causes a maximum error of $15 \mathrm{meV}(-8 \mathrm{meV})$ with BLYP and even smaller errors for the two other functionals.

The above tests establish that an inaccurate description of bond stretching is the main origin of the error in $E_{1 b}$ for the GGAs. Returning to the structures taken from the liquid we therefore plot in Fig. 4(b) the $E_{1 b}$ error against the length of the longest $\mathrm{O}-\mathrm{H}$ bond for each monomer. As with the systematic deformations of the equilibrium monomer, the errors in $E_{1 b}$ increase almost linearly with the $\mathrm{O}-\mathrm{H}$ bond length for the GGAs and for PBE0 they remain very close to zero except at the longest distances. Thus it can be inferred that monomers inside liquid water are energetically too easy to stretch for both PBE and BLYP. We note that a careful series of tests taking water clusters from a BLYP MD simulation along with subsequent tests with $\operatorname{CCSD}(\mathrm{T})$ established that none of the conclusions arrived at here are altered if BLYP structures are used [62].

Before moving on to the dimers, we point out that the discrepancies established here between the three xc functionals and $\operatorname{CCSD}(\mathrm{T})$ correlate well with the errors in the computed harmonic vibrational frequencies of an isolated water monomer (Table 【). Specifically for the two stretching frequencies PBE and BLYP are $\sim 115$ to $\sim 160 \mathrm{~cm}^{-1}(\sim 3-4 \%)$ softer than $\operatorname{CCSD}(\mathrm{T})$ (Table I), whereas, PBE0 is only $\sim 45 \mathrm{~cm}^{-1}(\sim 1 \%)$ harder. This one to one correspondence between error in harmonic vibrational frequencies and $E_{1 b}$ may also
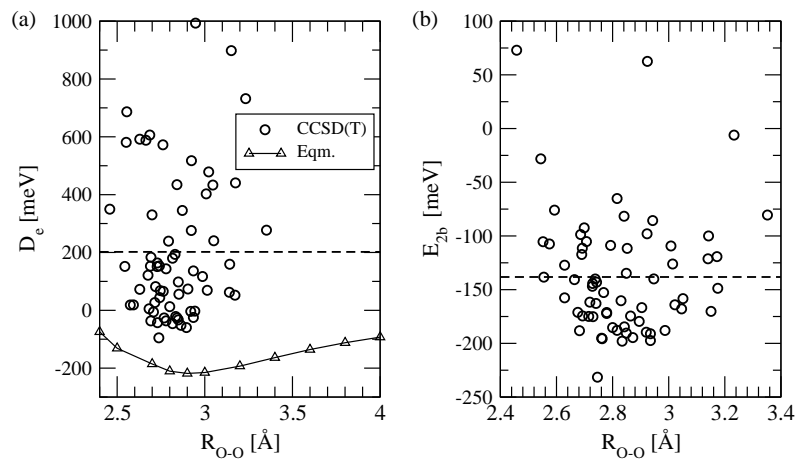

FIG. 5: (a) $\operatorname{CCSD}(\mathrm{T})$ total dissociation energies $\left(D_{e}\right)$ of the water dimers taken from the PBE liquid water simulation and the dissociation energy curve for a fully optimized gas phase dimer (Eqm.) as a function of the O-O distance $\left(R_{O-O}\right)$; (b) $\operatorname{CCSD}(\mathrm{T})$ two-body energies $\left(E_{2 b}\right)$ as a function of $R_{O-O}$ for the same dimers. The dashed lines represent the mean values of $D_{e}$ and $E_{2 b}$ in panels (a) and (b), respectively.

hold for other xc functionals and may therefore provide a cheap diagnostic to estimate in advance how reliably an xc functional will be at the determination of $E_{1 b}$.

\section{B. Dimers}

Now we move to the dimers extracted from the liquid, discussing first what $\operatorname{CCSD}(\mathrm{T})$ reveals about the stability of the dimers and then considering how well the three functionals perform. In Fig. 5 (a) the $\operatorname{CCSD}(\mathrm{T})$ dissociation energies are plotted as a function of the O-O distance within each dimer. Also reported is the equilibrium (i.e., fully optimized) $\operatorname{CCSD}(\mathrm{T})$ dissociation energy curve for a gas phase water dimer. As expected the equilibrium dimer binding energy curve provides a lower bound for the dissociation energies of the deformed dimers, which at each particular value of $R_{O-O}$ exhibit a range of values reflecting the range of dimer structures in the liquid. More importantly, Fig. 5(a) provides an overview of the range of dissociation energies for water dimers found inside the first coordination shell of PBE liquid water. The range is large: from -95 to $+993 \mathrm{meV}$, with the mean value being $+201 \mathrm{meV}$. Indeed $75 \%$ of the dissociation energies are positive, i.e., $75 \%$ of the dimers are unbound compared to two gas phase equilibrium water monomers. Upon decomposing the dissociation energies into the oneand two-body contributions we find that the average $E_{1 b}$ is $339 \mathrm{meV}$ and the average $E_{2 b}$ is $-137 \mathrm{meV}$ (Table 【I). Note that the average value of $E_{1 b}$ for the dimers is, of course, about twice $E_{1 b}$ for the monomers discussed above. Also note that this is a considerably larger $E_{1 b}$ than for the gas phase equilibrium water dimer, which is only $10 \mathrm{meV}$ (Table II). The two-body energy gives the binding between the water molecules and $97 \%$ of the dimers have an attractive $E_{2 b}$ [Fig. [5(b)]. The average value of $E_{2 b}$ at $-137 \mathrm{meV}$ is somewhat smaller than the corresponding value for the gas phase equilibrium dimer 
TABLE II: Mean values of the one-body, two-body, and dissociation energies of the deformed dimers (selected from the liquid) and the corresponding values for the gas phase equilibrium dimer with CCSD(T), PBE, BLYP, and PBE0. The differences between each DFT xc functional and $\operatorname{CCSD}(\mathrm{T}) / \mathrm{CBS}$ are given in parenthesis. Energies are in meV.

\begin{tabular}{cccc|ccc}
\hline \hline & \multicolumn{3}{c|}{ deformed } & \multicolumn{3}{c}{ Gas phase equilibrium } \\
\hline & $\mathrm{D}_{e}\left(\Delta \mathrm{D}_{e}\right)$ & $\mathrm{E}_{1 b}\left(\Delta \mathrm{E}_{1 b}\right)$ & $\mathrm{E}_{2 b}\left(\Delta \mathrm{E}_{2 b}\right)$ & $\mathrm{D}_{e}\left(\Delta \mathrm{D}_{e}\right)$ & $\mathrm{E}_{1 b}\left(\Delta \mathrm{E}_{1 b}\right)$ & $\mathrm{E}_{2 b}\left(\Delta \mathrm{E}_{2 b}\right)$ \\
\hline $\mathrm{CCSD}(\mathrm{T})$ & 201.9 & 339.2 & -137.2 & -211.6 & 9.7 & -221.4 \\
$\mathrm{PBE}$ & $121.9(80.0)$ & $268.0(71.2)$ & $-146.1(8.8)$ & $-219.9(8.3)$ & $3.7(6.0)$ & $-223.6(2.2)$ \\
$\mathrm{BLYP}$ & $159.3(42.6)$ & $254.4(84.8)$ & $-95.1(-42.1)$ & $-178.7(-32.9)$ & $2.4(7.3)$ & $-181.1(-40.3)$ \\
PBE0 & $200.7(1.2)$ & $346.1(-6.9)$ & $-145.3(8.1)$ & $-213.5(1.9)$ & $9.7(0.0)$ & $-223.2(1.8)$ \\
\hline \hline
\end{tabular}
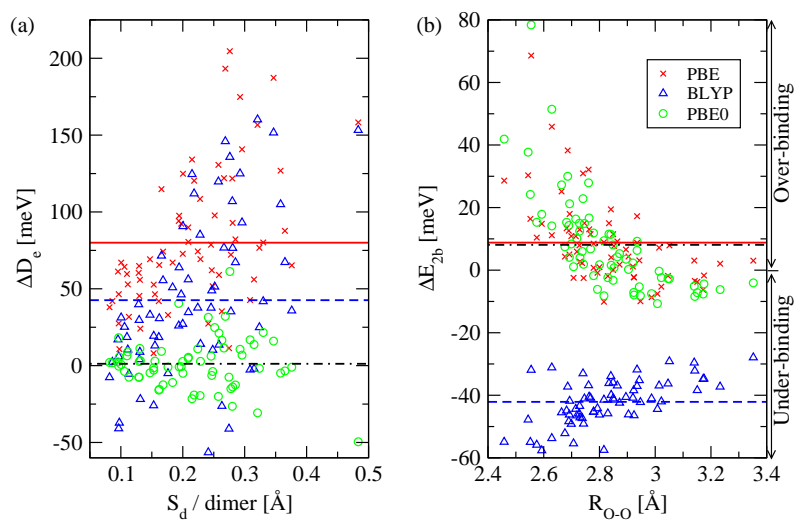

FIG. 6: (Color online) (a) Errors in dimer dissociation energy $\left(\Delta D_{e}\right)$ as a function of the sum of the deformation of the two monomers within each dimer for PBE, BLYP, and PBE0. (b) Errors in the two-body energy $\left(\Delta E_{2 b}\right)$ from PBE, $\mathrm{BLYP}$, and PBE0 as a function of the O-O distance $\left(R_{O-O}\right)$ within the dimers. Horizontal solid, dashed, and dash-dotted lines represent the average errors of PBE, BLYP, and PBE0, respectively.

of $-221 \mathrm{meV}$. Since the total dissociation energy for the dimers is just the sum of $E_{1 b}$ and $E_{2 b}$, it is quite obvious therefore that $E_{1 b}$ plays the major role in destabilizing the dimers.

Coming back to the performance of the xc functionals, Fig. 6(a) reports the error in the dissociation energies for each dimer. It can be seen that overall PBE0 performs very well, yielding an average error of $1 \mathrm{meV}$. BLYP and $\mathrm{PBE}$, on the other hand, yield quite large average errors of 43 and $80 \mathrm{meV}$, respectively. This behavior differs significantly from how these two functionals perform for the gas phase equilibrium water dimer, where errors of only -33 and $8 \mathrm{meV}$ are obtained (Table II). Thus we arrive at a key result, the performance of the two GGA functionals for the deformed dimer structures is inferior to what it is for the gas phase equilibrium dimer, with both functionals substantially overbinding the dimers taken from the liquid. Table II reports the key quantities that allow us to understand these results and why they contrast to the equilibrium gas phase dimers. As one might anticipate from section III A, the key is the one-body deformation energy. In the gas phase equilibrium dimer the absolute value of $E_{1 b}$ is small $(10 \mathrm{meV})$ and the resultant errors even smaller (Table II). Thus the performance of a functional for the gas phase equilibrium dimer is dominated by $E_{2 b}$, which is accurately described with $\mathrm{PBE}$ and PBE0 (8 and $2 \mathrm{meV}$ errors, respectively) and underestimated by some $33 \mathrm{meV}$ with BLYP. However, as we have seen in the structures taken from the liquid, $E_{1 b}$ is large $[339 \mathrm{meV}$ from $\operatorname{CCSD}(\mathrm{T})]$ and the associated errors from the GGA xc functionals become significant. Specifically, since both BLYP and PBE predict that the onebody deformation energy is much too small and predict either too weak or about right two-body energies then the total dissociation energies come out too large. BLYP proves to be more accurate than PBE simply because of more favorable cancelations of errors in $E_{1 b}$ and $E_{2 b}$ (Table II). Since BLYP is generally considered to produce too weak HBs between water molecules [38, 41, 44, 45] the overbinding observed here is remarkable. The obvious relevance of this finding to liquid water will be discussed below.

A characteristic feature of $\mathrm{HBs}$ between water molecules is that the covalent $\mathrm{O}-\mathrm{H}$ bonds of the donor molecules $\left(R_{\mathrm{O}-\mathrm{H}_{d}}\right)$ are elongated [63]. The elongation is a result of charge transfer from the acceptor water molecule to the $\mathrm{O}-\mathrm{H} \sigma^{*}$ antibonding orbital of the donor molecule [64, 65]. Since we find that it is too easy to stretch an $\mathrm{O}-\mathrm{H}$ bond with the GGAs, one can anticipate that this will further influence the strength of the HBs formed and in particular $E_{2 b}$. To investigate this we return to the gas phase equilibrium water dimer as a test case and systematically stretch the $\mathrm{O}-\mathrm{H}_{d}$ bond whilst keeping all other atoms fixed. Fig. 7(a) plots the change in $E_{2 b}$ as a function of the $\mathrm{O}-\mathrm{H}_{d}$ bond length with $\operatorname{CCSD}(\mathrm{T})$ and the three xc functionals. Clearly all methods predict that as the $\mathrm{O}-\mathrm{H}_{d}$ bond increases so too does $E_{2 b}$. However, all three xc functionals predict too rapid an increase compared to $\operatorname{CCSD}(\mathrm{T})$. This is best seen by the inset in Fig 77(a) which displays the error in the change of $E_{2 b}$ as a function of $R_{\mathrm{O}-\mathrm{H}_{d}}$ compared to $\operatorname{CCSD}(\mathrm{T})$. Likewise, $E_{2 b}$ increases slightly too rapidly with the three xc functionals for the dimers extracted from the liquid [Fig. 7(b)]; this is particularly apparent for PBE and PBE0. Thus in addition to it being too easy to stretch an $\mathrm{O}-\mathrm{H}$ bond with BLYP and $\mathrm{PBE}$, for 

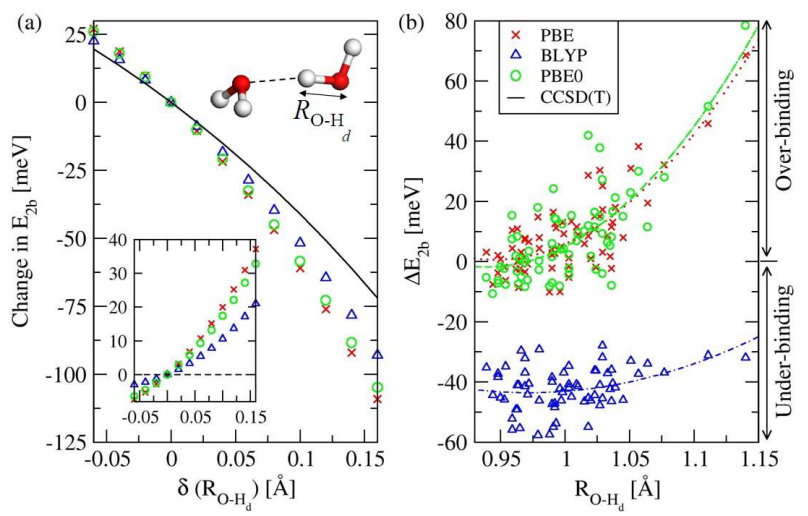

FIG. 7: (Color online) (a) Compared to the gas phase equilibrium dimer, the change in the two-body energy $\left(E_{2 b}\right)$ obtained from the systematic variation of the covalent $\mathrm{O}-\mathrm{H}$ bond of the donor 'H' atom $\left[\delta\left(R_{\mathrm{O}-\mathrm{H}_{d}}\right)\right]$ keeping all other atoms fixed. The inset shows the difference between the DFT xc functionals and $\operatorname{CCSD}(\mathrm{T})$. (b) Error in the two-body $\left(E_{2 b}\right)$ energy from DFT compared to CCSD(T) as a function of the $R_{\mathrm{O}-\mathrm{H}_{d}}$, obtained from the dimers from liquid water. Here positive values refer to a stronger two-body interaction. The dashed, dotted, and dash-dotted lines are quadratic fits to the PBE0, PBE, and BLYP data, respectively.

all three xc functionals the magnitude of the change in $E_{2 b}$ upon stretching is too great, further contributing to the overbinding of dimers with long $\mathrm{O}-\mathrm{H}_{d}$ bonds.

\section{Discussion}

It is clear from the last section that, despite the $E_{2 b}$ errors for the longest $\mathrm{O}-\mathrm{H}_{d}$ bonds, the overall performance of PBE0 is superior to that of the GGAs. To understand the origin of the difference between PBE and PBE0 the variation in the exchange and correlation energies was examined upon going from the gas phase equilibrium to the deformed water monomer structures (Table III). The variations in DFT exchange and correlation energies are then compared with the full HF exact exchange and $\operatorname{CCSD}(\mathrm{T})$ correlation. We note that the physical interpretation of exchange and correlation differs from DFT (PBE) to $\operatorname{CCSD}(\mathrm{T})$ and so use the data reported in Table III and Fig. 8 merely in the hope of obtaining some general qualitative insight. The basic finding from $\operatorname{CCSD}(\mathrm{T})$ is that upon going from the gas phase equilibrium to the deformed monomers there is a gain in the (negative) correlation energy and a loss in exchange energy [Fig. 8(a),(b)]. Naturally, the absolute change in the exchange energy is far greater than that in the correlation energy. The two DFT xc functionals predict a loss in the exchange energy but in contrast to $\operatorname{CCSD}(\mathrm{T})$ also a loss in the correlation energy, i.e., there is less correlation with $\mathrm{PBE}$ in the deformed monomers compared to the equilibrium monomer in the gas phase. Thus, in terms of the correlation energy, $\mathrm{PBE} / \mathrm{PBE} 0$ predicts qualitatively different behavior from
TABLE III: Average differences in the exchange and correlation contributions between the deformed monomers extracted from liquid water and the gas phase equilibrium monomer structure, obtained with $\mathrm{CCSD}(\mathrm{T}), \mathrm{PBE}$, and PBE0. Note that the exchange contribution of $\operatorname{CCSD}(\mathrm{T})$ refers to HF exact exchange. Values in parenthesis are the differences between the two xc functionals and $\operatorname{CCSD}(\mathrm{T})$. Here positive and negative values indicate energy loss and gain, respectively. All values are in $\mathrm{meV}$.

\begin{tabular}{cccc}
\hline \hline & $\mathrm{CCSD}(\mathrm{T})$ & $\mathrm{PBE}$ & $\mathrm{PBE} 0$ \\
\hline Correlation & -65.5 & $+35.2(-99.7)$ & $+34.5(-99.0)$ \\
Exchange & +825.6 & $+681.9(+143.7)$ & $+722.7(+102.9)$ \\
\hline Total & 761.1 & $717.1(+44.0)$ & $757.2(+3.9)$ \\
\hline \hline
\end{tabular}

$\operatorname{CCSD}(\mathrm{T})$ upon bond stretching. However, the missing correlation in the deformed monomers is compensated for by differences from $\operatorname{CCSD}(\mathrm{T})$ in the exchange energy. In PBE0, which predicts exchange energies in better agreement with HF (CCSD(T)) [Fig. 8(b)], the missing correlation is compensated by missing exchange so that overall the total energy changes are, as we have seen, very similar for PBE0 and CCSD(T). In PBE, however, the lack of correlation is not sufficient to compensate for the larger underprediction of the exchange energy [Fig. [8(b)]. Thus we find that PBE0 is superior to PBE simply because of a more favorable cancelation of the differences of exchange and correlation from $\operatorname{CCSD}(\mathrm{T})[\mathrm{Fig} .8(\mathrm{c})]$.

More generally the poor description of covalent $\mathrm{O}-\mathrm{H}$ bond stretching observed here with PBE and BLYP is likely to apply to many other GGAs. For example, tests with RPBE, mPWLYP, and BP86 (on the 92 monomers taken from our MD simulation) all produce one-body energies that are $30-40 \mathrm{meV}$ smaller than $\operatorname{CCSD}(\mathrm{T})$. Similarly, as with PBE0, the hybrid functionals B3LYP and $\mathrm{X} 3 \mathrm{LYP}$ predict rather accurate one-body energies, coming within $10 \mathrm{meV}$ of $\operatorname{CCSD}(\mathrm{T})$. Of course the conclusion reached here that HF exact exchange is necessary for the proper description of covalent bond stretching is consistent with what has long been known in the context of covalent bond breaking (and transition state energies) in the gas phase (see e.g. Refs. [66, 67, 68, 69, 70]).

Several previous studies have examined how DFT $\mathrm{xc}$ functionals perform in treating HBs between water molecules in water clusters $26,38,41,442,43,44,44,446$, 47, 48, 49, 50, 51]. Our study here is somewhat unconventional in that we have examined structures extracted directly from a liquid water simulation instead of exploring equilibrium gas phase structures. This has revealed significant differences in how PBE and BLYP perform for the structures extracted from the liquid compared to the known performance of these functionals for equilibrium gas phase water clusters. Thus we see that the behavior of these functionals for e.g. the gas phase equilibrium water dimer is not a good indicator for how these functionals perform for dimers extracted from the liquid. One must always exercise caution in making connections 

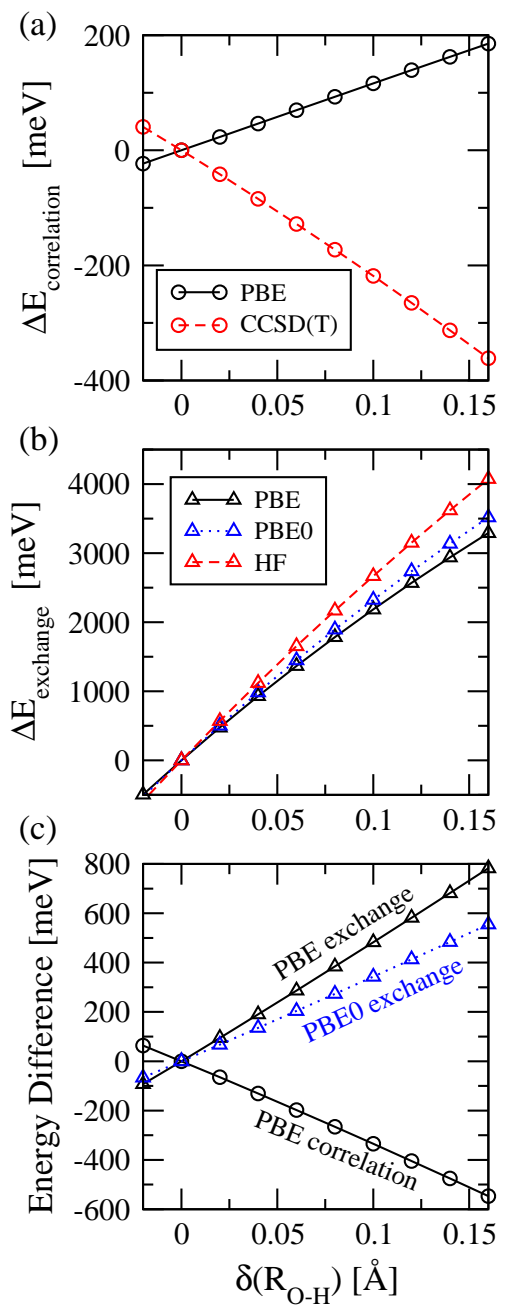

FIG. 8: (Color online) Variation in (a) correlation energy $\left(\Delta E_{\text {correlation }}\right)$ and (b) exchange energy $\left(\Delta E_{\text {exchange }}\right)$ with O$\mathrm{H}$ bond length for a gas phase water molecule. (c) Variation in the difference of DFT correlation and exchange energies in comparison to the $\operatorname{CCSD}(\mathrm{T})$ correlation and HF exact exchange energies, respectively, as a function of the monomer $\mathrm{O}-\mathrm{H}$ bond length. Positive and negative values refer to energy loss and gain, respectively.

between interaction energies of gas phase clusters and RDFs for the corresponding liquid phase, particularly in the present circumstances where we have only considered one- and two-body terms. Nonetheless, it is plausible that the overbinding observed here for PBE and BLYP, which originates in $E_{1 b}$ errors, is connected with the overstructuring of these functionals for liquid water. Indeed, because of the greater error cancelations between the one- and two-body energies calculated with BLYP, the overbinding of the dimers from the liquid is less for BLYP than for PBE. This may again provide an explanation for why $g_{\mathrm{O}-\mathrm{O}}^{\max }$ is less in BLYP compared to PBE. This thinking may also explain the low value of $g_{\mathrm{O}-\mathrm{O}}^{\max }$ reported in MD simulations with the RPBE functional $[19,21]$. A computed $\sim 50 \mathrm{meV}$ underestimation of the
$E_{2 b}$ for a gas phase equilibrium water dimer suggests that the likely errors in $E_{1 b}$ for this non-hybrid GGA will be more than compensated for. In addition, our results are consistent with and help to explain the results from the rigid water MD simulations [53, 54]. First, by fixing $\mathrm{O}-\mathrm{H}$ bonds at or close to the gas phase monomer equilibrium $\mathrm{O}-\mathrm{H}$ bond length, the $E_{1 b}$ error is eliminated or greatly reduced. Second, the large $E_{2 b}$ error associated with the longest $\mathrm{O}-\mathrm{H}_{d}$ bonds is also obviated.

Water molecules in other environments such as those in bulk ice or larger gas phase clusters will also possess deformed monomers with elongated bonds. These deformations are smaller than in liquid water but the effect is not negligible. For example, the average deformation of the monomers in a water hexamer is $0.05 \AA$ with PBE optimized geometries and in bulk ice Ih PBE predicts an average deformation of $\sim 0.06 \AA$. Based on the approximate relation between $E_{1 b}$ error and deformation established in Fig. 3, such deformations as encountered in small clusters and ice are likely to lead to errors in $E_{1 b}$ of $\sim 30-40 \mathrm{meV}$.

Finally, we point out that the suggestion that too facile bond stretching may result in an overstructured liquid is likely to be of relevance to other associated liquids apart from water. The relevant experimental and theoretical RDFs of other associated liquids are not as well established as liquid water. However, there are indications of BLYP simulations yielding overstructured RDFs for e.g. liquid ammonia [71, 72] and methanol 73, 74 despite BLYP underestimating the strength of the corresponding gas phase dimers by $\sim 45 \%$ compared to $\operatorname{CCSD}(\mathrm{T})$ [4].

\section{Summary}

In summary, from a PBE simulation of liquid water, monomers and bonded dimers (from the first coordination shell of the O-O RDF) were extracted. With $\operatorname{CCSD}(\mathrm{T}) 75 \%$ of the dimers were shown to be unbound compared to two gas phase equilibrium water monomers. This is mainly because the structures of the water monomers inside the liquid differ significantly from an equilibrium gas phase monomer. Indeed, with $\operatorname{CCSD}(\mathrm{T})$ we find that the average monomer extracted from the PBE liquid is about $150 \mathrm{meV}$ less stable than an equilibrium gas phase water monomer. Among the three xc functionals tested, the two GGAs (BLYP and PBE) underestimate the energy cost for monomer deformation (i.e., $E_{1 b}$ ) and as a consequence BLYP and PBE predict dissociation energies that are too large by 80 and 43 $\mathrm{meV}$, respectively, compared to $\operatorname{CCSD}(\mathrm{T})$. This is inferior to the performance of these functionals for the equilibrium water dimer and other water clusters in the gas phase. Overall PBE0 yields much more accurate dimer dissociation energies, mainly because it is not susceptible to such large bond stretching errors as the GGAs are. However, PBE0 is not free from deficiencies in treating the dimers examined here. Specifically, like 
the two other functionals, it predicts an increasing error in $E_{2 b}$ for the longest $\mathrm{O}-\mathrm{H}_{d}$ bonds. Finally, we have discussed the possible relevance of these results to DFT simulations of liquid water, to water in other environments, and to other associated liquids. In particular, we have suggested that the overbinding identified here may provide an explanation for the overstructured RDFs observed in BLYP and PBE simulations of liquid water. However, more work is required to further test this suggestion, with e.g. larger clusters that give access to higher order terms in the many-body decomposition and/or clusters embedded in an external electrostatic field that mimics the remaining water present in the liquid.

\section{Acknowledgments}

This work is supported by the European Commission through the Early Stage Researcher Training Network MONET, MEST-CT-2005-020908 (www.sljus.lu.se/monet). B.S. is grateful to Martin Fuchs for many helpful discussions. A.M's work is supported by a EURYI award (www.esf.org/euryi), the EPSRC, and the European Research Council.
[1] J. P. Perdew, K. Burke, and M. Ernzerhof, Phys. Rev. Lett. 77, 3865 (1996).

[2] A. D. Becke, Phys. Rev. A 38, 3098 (1988).

[3] W. Lee, C. Yang and R. G. Parr, Phys. Rev. B 37, 785 (1988).

[4] J. C. Grossman, E. Schwegler, E. W. Draeger, F. Gygi, and G. Galli, J. Chem. Phys. 120, 300 (2004).

[5] E. Schwegler, J. C. Grossman, F. Gygi, and G. Galli, J. Chem. Phys. 121, 5400 (2004).

[6] M. V. Fernández-Serra and E. Artacho, J. Chem. Phys. 121, 11136 (2004).

[7] M. J. McGrath, J. I. Siepmann, I.-F. W. Kuo, C. J. Mundy, J. VandeVondele, J. Hutter, F. Mohamed, and M. Krack, ChemPhysChem 6, 1894 (2005).

[8] M. J. McGrath, J. I. Siepmann, I.-F. W. Kuo, and C. J. Mundy, Molec. Phys. 104, 3619 (2006).

[9] M. J. McGrath, J. I. Siepmann, I.-F. W. Kuo, C. J. Mundy, J. VandeVondele, J. Hutter, F. Mohamed, and M. Krack, J. Phys. Chem. A 110, 640 (2006).

[10] H.-S. Lee and M. E. Tuckerman, J. Phys. Chem. A 110, 5549 (2006).

[11] H.-S. Lee and M. E. Tuckerman, J. Chem. Phys. 125, 154507 (2006).

[12] H.-S. Lee and M. E. Tuckerman, J. Chem. Phys. 126, 164501 (2007).

[13] T. Todorova, A. P. Seitsonen, J. Hutter, I.-F. W. Kuo, and C. J. Mundy, J. Phys. Chem. B 110, 3685 (2006).

[14] J. VandeVondele, F. Mohamed, M. Krack, J. Hutter, M. Sprik, and M. Parrinello, J. Chem. Phys. 122, 014515 (2005).

[15] M. Guidon, F. Schiffmann, J. Hutter, and J. VandeVondele, J. Chem. Phys. 128, 214104 (2008).

[16] T. D. Kühne, M. Krack, and M. Parrinello, J. Chem. Theory Comput. 5, 235 (2009).

[17] P. H.-L. Sit and N. Marzari, J. Chem. Phys. 122, 204510 (2005).

[18] I.-C. Lin, A. P. Seitsonen, M. D. Coutinho-Neto, I. Tavernelli, and U. Rothlisberger, J. Phys. Chem. B 113, 1127 (2009).

[19] M. V. Fernández-Serra, G. Ferlat, and E. Artacho, Molecular Simualtion 31, 361 (2005).

[20] D. Asthagiri, L. R. Pratt, and J. D. Kress, Phys. Rev. E 68, 041505 (2003).

[21] A. E. Mattson and T. R. Mattson, J. Chem. Theory Comput. 5, 887 (2009).
[22] I.-F. W. Kuo, C. J. Mundy, M. J. McGrath, J. I. Siepmann, J. VandeVondele, M. Sprik, J. Hutter, B. Chen, M. L. Klein, F. Mohamed, et al., J. Phys. Chem. B 108, 12990 (2004).

[23] M. Sprik, J. Hutter, and M. Parrinello, J. Chem. Phys. 105, 1142 (1996).

[24] P. L. Silvestrelli and M. Parrinello, J. Chem. Phys. 111, 3572 (1999).

[25] S. Yoo, X. C. Zeng, and S. S. Xantheas, J. Chem. Phys. 130, 221102 (2009).

[26] B. Santra, A. Michaelides, M. Fuchs, A. Tkatchenko, C. Filippi, and M. Scheffler, J. Chem. Phys. 129, 194111 (2008).

[27] B. Chen, I. Ivanov, M. L. Klein, and M. Parrinello, Phys. Rev. Lett. 91, 215503 (2003).

[28] G. S. Fanourgakis, G. K. Schenter, and S. S. Xantheas, J. Chem. Phys. 125, 141102 (2006).

[29] F. Paesani, S. Luchi, and G. A. Voth, J. Chem. Phys. 127, 074506 (2007).

[30] C. J. Burnham, D. J. Anick, P. K. Mankoo, and G. F. Reiter, J. Chem. Phys. 128, 154519 (2008).

[31] J. A. Morrone and R. Car, Phys. Rev. Lett. 101, 017801 (2008).

[32] S. B. Rempe, T. R. Mattson, and K. Leung, Phys. Chem. Chem. Phys. 10, 4685 (2008).

[33] R. Car and M. Parrinello, Phys. Rev. Lett. 55, 2471 (1985).

[34] K. Laasonen, M. Sprik, M. Parrinello, and R. Car, J. Chem. Phys. 99, 9080 (1993).

[35] A. K. Soper, Chem. Phys. 258, 121 (2000).

[36] T. Head-Gordon and M. E. Johnson, Proc. Nat. Acad. Sci. U.S.A 103, 7973 (2006).

[37] A. K. Soper, J. Phys. Condens. Matter 19, 335206 (2007).

[38] B. Santra, A. Michaelides, and M. Scheffler, J. Chem. Phys. 127, 184104 (2007).

[39] E. E. Dahlke, R. M. Olson, H. R. Leverentz, and D. G. Truhlar, J. Phys. Chem. A 112, 3976 (2008).

[40] Y. Zhao and D. G. Truhlar, J. Chem. Theory Comput. 1, 415 (2005).

[41] X. Xu and W. A. Goddard III, J. Phys. Chem. A 108, 2305 (2004).

[42] J. T. Su, X. Xu, and W. A. Goddard III, J. Phys. Chem. A 108, 10518 (2004).

[43] J. Ireta, J. Neugebauer, and M. Scheffler, J. Phys. Chem. A 108, 5692 (2004). 
[44] S. Tsuzuki and H. P. Lüthi, J. Chem. Phys. 114, 3949 (2001).

[45] J. J. Novoa and C. Sosa, J. Phys. Chem. 99, 15837 (1995).

[46] S. S. Xantheas, J. Chem. Phys. 102, 4505 (1994).

[47] J. A. Anderson and G. S. Tschumper, J. Phys. Chem. A 110, 7268 (2006).

[48] G. C. Shields and K. N. Kirscner, Synthesis and Reactivity in Inorganic, Metal-Organic, and Nano-Metal Chemistry 38, 32 (2008).

[49] A. Csonka, G. I. Ruzsinszky and J. P. Perdew, J. Phys. Chem. B 109, 21471 (2005).

[50] E. E. Dahlke and D. G. Truhlar, J. Phys. Chem. B 109, 15677 (2005).

[51] E. E. Dahlke and D. G. Truhlar, J. Phys. Chem. B 110, 10595 (2006).

[52] K. Kim and K. D. Jordan, J. Phys. Chem. 98, 10089 (1994).

[53] M. Allesch, E. Schwegler, F. Gygi, and G. Galli, J. Chem. Phys. 120, 5192 (2004).

[54] K. Leung and S. B. Rempe, Phys. Chem. Chem. Phys. 8, 2153 (2006).

[55] C. Adamo and V. Barone, J. Chem. Phys. 110, 6158 (1999).

[56] J. Hutter et al., computer code CPMD, version 3.11; Copyright IBM Corp. 1990-2006, Copyright für Festkörperforschung Stuttgart, Germany, 1997-2001, http://www.cpmd.org/

[57] S. Goedecker, M. Teter, and J. Hutter, Phys. Rev. B 54, 1703 (1996).

[58] M. J. Frisch et al., Gaussian 03, Revision C.02, Gaussian, Inc., Wallingford CT, 2004.

[59] R. A. Kendall, T. H. Dunning, Jr., and R. J. Harrison, J. Chem. Phys. 96, 6796 (1992); T. H. Dunning, Jr., D. Woon, and K. A. Peterson (unpublished).

[60] E. J. Bylaska et al., "NWChem, A Computational Chemistry Package for Parallel Computers, Version 5.0" (2006), Pacific Northwest National Laboratory, Richland, Washington 99352-0999, USA.
[61] The choice of a PBE optimized monomer as the equilibrium monomer reference does not influence the general conclusions arrived at here. For example, if a $\operatorname{CCSD}(\mathrm{T})$ optimized monomer reference is used, the DFT $E_{1 b}$ errors on average differ by no more than $5 \mathrm{meV}$ from those reported.

[62] To check if the computed errors in $E_{1 b}$ from the xc functionals are sensitive to the functional used in the original MD simulation a separate 30 ps BLYP MD simulation was performed. From the BLYP simulation 12 monomers were extracted and average errors in $E_{1 b}$ of 23 (PBE), 27 (BLYP), and $-0.3 \mathrm{meV}$ (PBE0) obtained, compared to average errors in $E_{1 b}$ of 32 (PBE), 38 (BLYP), and -3 $\mathrm{meV}(\mathrm{PBE} 0)$ as obtained from the PBE MD simulation reported.

[63] G. A. Jeffrey, An Introduction to Hydrogen Bonding (Oxford University Press, Inc., New York, 1997).

[64] K. Müller-Dethlefs and P. Hobza, Chem. Rev. 100, 143 (2000).

[65] R. Z. Khailiullin, A. T. Bell, and M. Head-Gordon, Chem. Eur. J. 15, 851 (2009).

[66] J. Baker, J. Andzelm, M. Muir, and P. R. Taylor, Chem. Phys. Lett. 237, 53 (1995).

[67] J. L. Durant, Chem. Phys. Lett. 256, 595 (1996).

[68] B. J. Lynch, P. L. Fast, M. Harris, and D. G. Truhlar, J. Phys. Chem. A 104, 4811 (2000).

[69] Y. Zhao, N. González-García, and D. G. Truhlar, J. Phys. Chem. A 109, 2012 (2005).

[70] B. G. Janesko and G. E. Scuseria, J. Chem. Phys. 128, 244112 (2008).

[71] M. Diraison and G. J. Martyna, J. Chem. Phys. 111, 1096 (1999).

[72] A. D. Boese, A. Chandra, J. M. L. Martin, and D. Marx, J. Chem. Phys. 119, 5965 (2003).

[73] M. Pagliai, G. Cardini, R. Righini, and V. Schettino, J. Chem. Phys. 119, 6655 (2003).

[74] J.-W. Handgraaf, E. J. Meijer, and M.-P. Gaigeot, J. Chem. Phys. 121, 10111 (2004). 